Evolution

\section{Complex vertebrate structures}

\section{John Maynard Smith}

IT is a safe prediction that, from time to time, one of the less illiterate newspapers (the Independent is the most recent example; see illustration) will carry a centre-page article arguing that darwinism must be wrong because the structure of living organisms is too complex to have arisen by chance, and because anything other than a perfect structure would be useless. The orthodox reply is that we know of no other mechanism that could explain the evolution of adapted structure, that there is no quantitative difficulty in accounting for the accumulation of the necessary genetic information by selection, and that an imperfect structure is often not useless: an image-forming eye is fine, but an organ that tells you whether the light is on, or where it is coming from, may be a lot better than nothing, at least if you are a flatworm. Is there anything more useful that can be said? A recent meeting on the evolution of complex structures in vertebrates* attempted to answer this question.

Three groups discussed, respectively, the evolution of locomotion, of feeding, and of viviparity, and a fourth debated the problem of evolutionary mechanisms in more general terms. Did any general principles emerge from the many facts about vertebrate function that were presented? Two themes arose repeatedly - the significance of 'coupling', and the role of phylogenetic information in testing hypotheses. The term coupling was used in (at least) two senses: it meant either that the same function requires the cooperation of two or more structures or that the same structure performs two different functions. Little need be said about the former meaning: for example, the evolution of mammalian from reptilian locomotion required changes, not only in the skeleton and muscles, but in the nervous, vascular and respiratory systems. The necessity for simultaneous changes in all these systems may help to explain the extreme slowness of evolution, which is such a puzzle to those of us familiar with the results of artificial selection. But the other type of coupling - one structure performing two functions - has some less obvious consequences.

The general thesis is as follows. If a structure performs two functions, it is likely to be conservative in evolution. If, for any reason, it is released from one of those functions, this is often followed by rapid evolutionary specialization, and

\footnotetext{
* Dahlem workshop on complex organismal functions, integration and evolution in vertebrates, West Berlin, 28 August-
} 2 September 1988 . Proceedings to be published by Wiley. sometimes diversification, for the other. An example will make this clear. In lungbreathing salamanders, the hyolingual skeleton is used as a pump to force air into the lungs. It is also used in feeding - in the aquatic larva to suck in water, and in the adult to propel the tongue in catching insects. However, loss of lungs has ocurred repeatedly in salamanders, and in many cases this has been followed by the evolution of a mechanism propelling the tongue to a greater distance, with greater velocity and precision. But there is still coupling between feeding and breathing in the aquatic larva. In the Bolitoglossine salamanders the aquatic larval stage has been lost, and further specialization of a high-speed projectile tongue has occurred: direct development has also made possible the evolution of stereopsis, needed to aim the tongue accurately.

A large-scale scheme based on the idea of coupling concerns the evolution of tetrapod locomotion. In a manner reminiscent of a recent US president, lizards cannot run and breathe at the same time, and hence are incapable of rapid sustained locomotion. This is because the rib-cage and associated musculature are used in both locomotion and breathing, but in different ways. The problem has been partially solved in the crocodiles by the evolution of an abdominal septum and diaphragmatic muscle used when breathing in. Birds can breathe and fly at the same time, but with different periodicities, so the two processes must be mechanically independent. This has been brought about by the evolution of a rigid rib-cage, of a rigid sternum to which the light muscles are attached, and of a novel machinery for driving air through the constant-volume lung. Mammals have taken a different road. In the canter, gallop and bipedal hop, there is a rigid 1:1 phase locking of gait and breathing. The locomotory muscles help to drive

respiration, as do the inertial movements of the liver, which is rigidly attached to the diaphragm. Thus the birds have solved the problem by uncoupling: they use different structures for locomotion and breathing. The mammals continue to use some of the same structures for both purposes, but the evolution of new gaits, which involve flexing the backbone in a vertical rather than a lateral plane, has led to a system in which the two processes help rather than hinder one another.

Is there any way in which such ideas can be tested? How do we test the idea that lung loss is a cause of feeding specialization in salamanders, or that the acquisition of an additional and separately movable jaw apparatus in Cichlid fishes led to their adaptive radiation? The first requirement one must make of any such hypothesis is that it be plausible on engineering grounds. But often the comparative data enable us to go further. Suppose our

\title{
New answers to the scientific quarrels with Darwinism
}

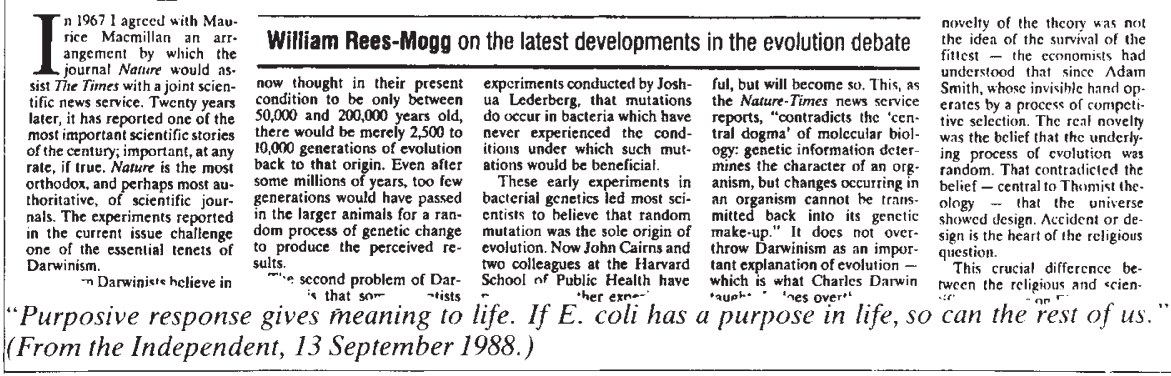

hypothesis is that trait $\mathrm{A}$ is responsible for the evolution of trait $B$. If we have phylogenetic information in the form of a cladogram, we can ask the following questions. Is it true that the appearance of $A$ preceded that of $B$ ? Has trait A arisen more than once, and, if so, was it always (or usually) followed by the appearance of $B$ ? The use of phylogenetic information in this way is still in its infancy, but, combined with appropriate statistical analysis of comparative data, it is spreading rapidly. This is admirable, but it is no substitute for engineering common sense. I do not need comparative data to tell me that a bird has a stout coracoid because, if it didn't, its shoulder joint would collapse onto its sternum every time its pectoral muscles contracted.

The group discussing evolutionary mechanisms was, inevitably, more argumentative than the others, and perhaps generated more heat than light. There was much discussion of development but, at a deep level, no real disagreement. No-one doubts that development limits the range of phenotypes that can arise in a particular taxon, and so constrains the course of evolution. What we need is more specific information about the nature of the constraints. 